\title{
A Pilot Project of Systematic Tuberculosis Screening in the Elderly in a South Korean Province
}

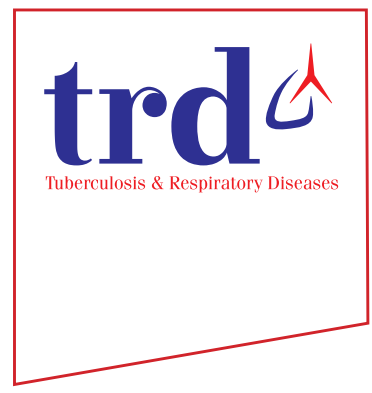

\author{
Hyunwoo Kim, M.P.H. ${ }^{1}{ }^{(\mathbb{D}}$, Hee-Jin Kim, M.D., M.P.H. ${ }^{2}$, Kyung-Hyun Oh, M.D., M.P.H. ${ }^{3}$, Hwan-Wook \\ Oh, B.P.H. ${ }^{4}$ and Hongjo Choi, M.D., Ph.D. ${ }^{1}$ (i) \\ ${ }^{1}$ Department of Research and Development, The Korean Institute of Tuberculosis, Korean National Tuberculosis Association, \\ Cheongju, ${ }^{2}$ The Korean Institute of Tuberculosis, Korean National Tuberculosis Association, Cheongju, ${ }^{3}$ Department of \\ Program Cooperation, The Korean Institute of Tuberculosis, Korean National Tuberculosis Association, Cheongju, ${ }^{4}$ Gwangju \\ Jeonnam Branch, Korean National Tuberculosis Association, Cheongju, Korea
}

Background: Tuberculosis (TB) is a major infectious disease in South Korea causing substantial disease burden, particularly in the elderly. This study aimed to identify the case detection rate of mobile TB screening for the elderly conducted in the Jeollanam-do region and to analyze risk factors of active TB.

Methods: We screened the elderly population ( $\geq 65$ years old) in Jeollanam-do from August to December 2017. Chest radiography was performed for all participants. Participants with TB presumptive signs were asked to submit sputum specimen(s). Sputum smear, culture, and polymerase chain reaction analyses were performed. Cascade analysis, chisquared tests, and Fisher exact tests were used to evaluate screening performance.

Results: In total, 12,402 participants were screened, and 211 (1.7\%) were suspected to have active TB; 181 of the suspected patients (85.8\%) underwent sputum smear test, and $16(8.8 \%)$ patients were confirmed to have TB. The TB prevalence among the elderly was bacteriologically confirmed to be 129 per 100,000 individuals, which was similar to national TB notification data for the same age groups. The proportion of active TB cases increased with age, and differed based on sex and past TB history. However, TB-related symptoms, comorbidity status, and TB screening history within 12 months were not predictive of active TB.

Conclusion: This study identified that the prevalence rate was similar to national TB notification data from the same age groups. Periodic, community-based, systematic TB screening among the elderly population is recommended.

Keywords: Tuberculosis; Thorax; Radiography; Pilot Projects; Mass Screening; Asian Continental Ancestry Group; Aged

Address for correspondence: Hongjo Choi, M.D., Ph.D.

Department of Research and Development, Korean Institute of

Tuberculosis, Korean National Tuberculosis Association, 168-5,

Osongsaengmyeong4-ro, Osong-eup, Heungdeok-gu, Cheongju 28158,

Korea

Phone: 82-43-249-4960, Fax: 82-43-249-4965

E-mail: hongjochoi@gmail.com

Received: Jul. 31, 2018

Revised: Dec. 13, 2018

Accepted: Jan. 14, 2019

Published online: Feb. 28, 2019

(c) It is identical to the Creative Commons Attribution Non-Commercial License (http://creativecommons.org/licenses/by-nc/4.0/).

Copyright @ 2019

The Korean Academy of Tuberculosis and Respiratory Diseases.

\section{Introduction}

Tuberculosis (TB) is the ninth leading cause of death worldwide; it ranked higher than human immunodeficiency virus/ acquired immune deficiency syndrome. According to the Global TB Report in 2017, the TB notification rate in 2016 was 6.3 million, but the estimated TB incidence was 10.4 million. Although the incidence and mortality rates have recently started to decrease, it will remain challenging to meet the $\mathrm{TB}$ targets by the end of 2030 unless the use of the current screening tools is maximized ${ }^{1}$. Global efforts have paid less attention to the elderly with TB. Most of the concerns regarding TB among the elderly, including the challenges in detecting and managing TB, have risen in developed countries ${ }^{2}$. However, 
considering the rapid aging trends worldwide, it will be challenging to achieve the global TB targets.

In South Korea, among all infectious diseases, TB has the highest prevalence and mortality rates and it causes intermediate disease burden ${ }^{3}$. According to the Annual Report on Notified TB in Korea in 2017, approximately $42 \%$ of all TB patients were aged $\geq 65$ years, of which $82 \%$ died owing to $\mathrm{TB}^{4}$. Similar problems have been reported in countries with a low TB incidence, including the United States, Japan, and the United Kingdom, as well as in those with a high TB incidence, including China and India ${ }^{5-9}$. The proportion of individuals newly infected with TB increased in the elderly population from 2001 to 2010, whereas the proportion of individuals newly infected with TB in other age groups has started to decline ${ }^{10}$. Additionally, according to the study conducted by Kim and $\mathrm{Yim}^{11}$, when the age group with the highest risk of TB becomes $\geq 65$ years of age in 2020 , the overall TB prevalence rate is likely to increase again and TB in the elderly could become an even greater issue in South Korea. Therefore, considering the epidemiological shifts including an increase in the percentage of elderly TB patients in Korea, it is necessary to prioritize the treatment of the elderly in the national TB control strategy. A spatial analysis was conducted to understand the regional differences in TB incidence; consequently, several regions with a high TB incidence in the elderly were identified ${ }^{12}$. As one of those provinces, the Jeollanam-do (JN) established a 3-year systematic TB screening project directed toward individuals aged $\geq 65$ years, thereby aiming to cover approximately $70 \%$ of the target group. Here, we report findings from the first-year pilot project started in 2017. The study aimed to identify the case detection rate of mobile TB screening for the elderly and risk factors of active TB. Additionally, we compared the prevalence of the study with national notification rate of the same age groups.

\section{Materials and Methods}

The JN regional government launched a project to systematically screen $70 \%$ of the eligible elderly population until 2020 . In 2017, as a pilot project, the systematic screening project planned to include 12,500 people from the designated regions. Among the administrative districts in JN, two small cities and 10 towns with a high incidence of $\mathrm{TB}$ were selected as the target areas for screening. Among those regions, several townships were designated the regions to be screened in 2017; the other regions were to be screened over the next 3 years. To investigate the elderly population aged $\geq 65$ years, the Korean National Tuberculosis Association (KNTA) collaborated with health centers in JN. TB screening was performed using a mobile examination vehicle, equipped with chest radiograph (X-ray) machines, from August to December 2017. Before the screening, the team educated participants about TB screen- ing and treatment and conducted a structured questionnaire among them. The questionnaire consisted of self-reported questions on: TB-related symptoms including cough and sputum presence and duration; previous X-ray screening history within 12 months; previous TB treatment history; other comorbidities. The project team informed the health center about the participants who displayed presumptive TB signs (i.e., they were presumed to have TB or had undetermined TB activity), or were determined to have active TB on chest radiography, and asked them to visit the health center for sputum collection. The chest radiography was interpreted by double reading (one pair of observers read the films independently) among three radiologists of the JN branch of KNTA as being normal or suspicious of $\mathrm{TB}$, or as having undetermined $\mathrm{TB}$ activity, active TB, inactive TB, or as suggestive of other diseases. Subsequently, sputum smear and culture analyses and/ or molecular diagnoses were performed. Smear, solid culture, and liquid culture tests were performed for all participants with presumptive TB signs. After the test, no further tests were performed for participants with smear positive results, and Xpert tests were performed for those with negative smear results (97.2\% among all cases with presumptive TB signs). Additional polymerase chain reaction tests were performed if negative results were obtained from the Xpert test $(96.7 \%$ among all cases with presumptive TB signs).

To evaluate the screening performance, first, we conducted cascade analysis as suggested by the World Health Organization $^{13}$. The number of patients successfully completing treatment is a final indicator in cascade analyses; however, treatment success rates were not reported in this study be-

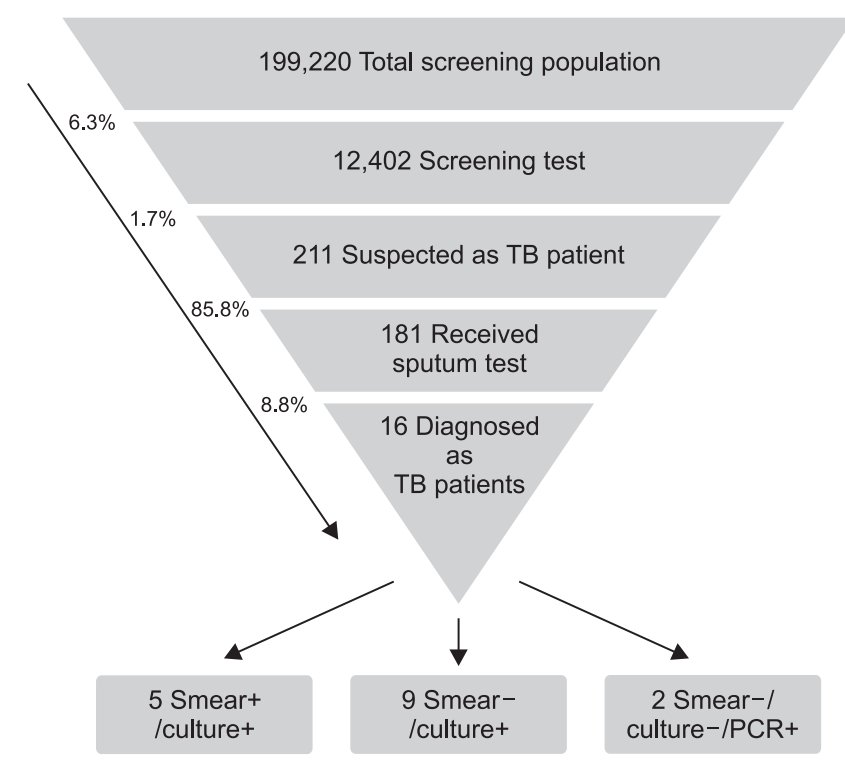

Figure 1. Cascade analysis of tuberculosis (TB) screening project involving the elderly who live in the Jeollanam-do region. PCR: polymerase chain reaction. 
cause this pilot screening project did not involve treatment follow-up for active TB patients. Second, TB prevalence was calculated from the screening project results and compared with previous surveillance data for the same age groups. For the comparison between the national notification rates and the prevalence within the project, we reanalyzed previous reports that used TB notification data in 2009 and 2014. These are cohort data and include patients at least 65 years of age, in whom active TB was bacteriologically confirmed as new drug-susceptible TB in 2009 and $2014^{14}$. This second analysis aimed to examine the demographic and clinical characteristics of the participants and verify whether there was a difference in TB prevalence based on these characteristics. Additionally, we compared the project outcomes with those of a previously conducted active case finding project (data not published) in the same region from 2014 to 2017. The JN branch of KNTA annually conducts systematic TB screening among vulnerable populations including the elderly, people living in long-term care facilities, and immigrants. The concept of the number needed to screen (NNS) was used to compare outcomes among the projects. We conducted chi-squared tests to determine the association between each characteristic and the detection of presumptive or active TB cases. If the sample size was too small (less than five) in a cell, Fisher's exact tests were used to test for associations instead. Stata 15 SE (developed by StataCorp., released by 2017 November 6) version was used for all statistical analyses. This study was reviewed by institutional review board at Korean National Tuberculosis Association (2018-KNTA-IRB-005) and informed consent was waived by institutional review board.

Table 1. Differences in the prevalence rates for cases suspected of TB and those diagnosed with TB depending on various demographic and clinical characteristics

\begin{tabular}{|c|c|c|c|c|c|}
\hline & \multirow{3}{*}{\begin{tabular}{|c|} 
Total \\
No. $(\%)$ \\
\end{tabular}} & \multicolumn{4}{|c|}{ Screening outcomes } \\
\hline & & \multicolumn{2}{|c|}{ Suspected } & \multicolumn{2}{|c|}{ Active TB } \\
\hline & & No. (\%) & p-value* & No. (\%) & p-value* \\
\hline \multicolumn{6}{|l|}{ Age, yr } \\
\hline $60-69$ & $2,832(22.8)$ & $19(0.67)$ & $<0.001$ & $1(0.04)$ & 0.039 \\
\hline $70-74$ & 2,709 (21.8) & $43(1.59)$ & & $1(0.04)$ & \\
\hline $75-79$ & $3,715(30.0)$ & $69(1.86)$ & & $5(0.13)$ & \\
\hline $80-84$ & 2,099 (16.9) & $46(2.19)$ & & $4(0.19)$ & \\
\hline$\geq 85$ & $1,047(8.4)$ & $34(3.25)$ & & $5(0.48)$ & \\
\hline \multicolumn{6}{|l|}{ Sex } \\
\hline Male & 4,205 (33.9) & $110(2.62)$ & $<0.001$ & $10(0.24)$ & 0.010 \\
\hline Female & $8,197(66.1)$ & $101(1.23)$ & & $6(0.07)$ & \\
\hline \multicolumn{6}{|l|}{ Symptom of TB } \\
\hline Yes & $792(6.4)$ & $28(3.54)$ & $<0.001$ & $3(0.38)$ & NS \\
\hline No & 11,608 (93.6) & $183(1.58)$ & & $13(0.11)$ & \\
\hline \multicolumn{6}{|l|}{ Screening history } \\
\hline Yes & 2,285 (18.4) & $39(1.71)$ & NS & $4(0.18)$ & NS \\
\hline No, unknown & 10,117 (81.6) & $172(1.70)$ & & $12(0.12)$ & \\
\hline \multicolumn{6}{|l|}{ TB history } \\
\hline Yes & $593(4.8)$ & $52(8.77)$ & $<0.001$ & $3(0.51)$ & 0.038 \\
\hline No, unknown & $11,809(95.2)$ & $159(1.35)$ & & $13(0.11)$ & \\
\hline \multicolumn{6}{|l|}{ Co-morbidities ${ }^{\dagger}$} \\
\hline Yes & $7,460(60.2)$ & $117(1.57)$ & NS & $7(0.09)$ & NS \\
\hline No & $4,942(39.8)$ & $94(1.90)$ & & $9(0.18)$ & \\
\hline
\end{tabular}

*p-values were calculated using chi-square tests. ${ }^{\dagger}$ Comorbidities among active TB cases: hypertension $(n=4)$, cardiovascular disease $(n=1)$, dyslipidemia $(\mathrm{n}=1)$, and gastrointestinal disease $(\mathrm{n}=1)$.

TB: tuberculosis; NS: not significant. 


\section{Results}

\section{Screening project results}

In the JN region, 12,508 people were screened; however, for 102 participants X-ray screening was not conducted as a result of their immobility or refusal to undergo screening. Thus, in total, 12,402 individuals were included in the analysis. This was equivalent to $6.3 \%$ of the total population in 12 designated small cities and towns $(\mathrm{n}=199,220)$. Of the people screened for TB, 211 (1.7\%) were identified with presumptive TB signs via chest X-ray scans and were therefore asked to undergo a sputum test. Among them, of the 181 participants (85.8\%) who agreed to undergo a sputum test, $16(8.8 \%)$ were diagnosed with active TB. Of those with active TB, five (31.3\%) were both smear- and culture-positive, nine (56.2\%) were smear-negative but culture-positive, and two (12.5\%) only tested positive using molecular tests (Figure 1). Therefore, the TB prevalence in the elderly, which was bacteriologically confirmed in the JN area through this pilot screening project, was 129 per 100,000 (95\% confidence interval, 79-209). The NNS in order to find an active TB case was 775 individuals.

\section{Demographic and clinical characteristics}

As shown in Table 1, a significant difference in the proportion of suspected TB cases among various age groups was noted, indicating an increasing trend with age $(\mathrm{p}<0.001)$. A similar trend was also noted among active TB cases; the proportion of elderly individuals aged $>85$ years diagnosed with TB was $0.48 \%$, which was 12 times greater than that of individuals aged $65-69$ years $(\mathrm{p}=0.039)$.

TB-related symptoms and TB screening history within 12 months were not associated with active TB diagnosis. Although a higher number of individuals displaying TB-related symptoms were identified as active TB patients than those without TB symptoms, the difference was not statistically significant ( $\mathrm{p}=0.196)$ (Table 1). Additionally, there was no as- sociation between TB case detection and prior screening for TB within the last 12 months.

Additionally, the data in Table 1 reveals that the proportion of males with active TB was significantly higher than that of females, and individuals with a history of TB were significantly more likely to have presumptive TB or confirmed, active TB. Interestingly, comorbidities were not significant predictors of the detection of active TB cases.

\section{Comparison with previous reports}

We compared the project outcomes with the national TB notification data among the same age groups. The prevalence rate in this study was 129 per 100,000, which was slightly higher than the previously reported notification rates among bacteriologically confirmed, elderly cases which were 105.5 per 100,000 and 117.7 per 100,000 in 2009 and 2014, respectively (Table 2). However, this difference did not significantly differ. In an additional comparison between the project outcomes and other screening efforts among other vulnerable groups in $\mathrm{JN}$, we found that our pilot project yielded a higher case detection rate and required a lower NNS (Table 3 ).

\section{Discussion}

To our knowledge, this is the first complete enumeration of TB only among the elderly as part of a community-based project in South Korea. In this project, we examined 12,402 patients and identified 16 patients with TB. TB prevalence in the elderly aged $\geq 65$ years in the JN region was 129 per 100,000 . Our findings were consistent with those from a previous report that used the TB reporting data from the Korea Centers for Disease Control and Prevention, and found that the notification rates for new drug-susceptible TB cases among the elderly aged $\geq 65$ years in 2009 and 2014 were approximately 106 and 118 per 100,000, respectively (Table 2$)^{14}$. Hence, the prevalence rates in our pilot project did not differ

Table 2. Comparison of the TB notification rate between the " 2009 and 2014 cohort of newly notified TB cases" and the "2017 TB screening for the elderly people living in the JN region"

\begin{tabular}{|c|c|c|c|}
\hline & \multicolumn{2}{|c|}{ National TB report data* } & \multirow{2}{*}{2017 TB screening for the elderly (JN) } \\
\hline & 2009 & 2014 & \\
\hline Study population & $5,267,708$ & $6,520,607$ & 12,402 \\
\hline TB cases per 100,000 & 105.5 & 117.7 & 129 \\
\hline Odds ratio ${ }^{\dagger}$ & 0.82 & 0.91 & Reference \\
\hline
\end{tabular}

*Choi HJ (2017). Development of a pilot model for TB management and care for socioeconomically vulnerable regions and groups. Cheongju: Korea Centers for Disease Control and Prevention ${ }^{14}{ }^{\dagger}$ Odds ratios were calculated using chi-square tests.

TB: tuberculosis; JN: Jeollanam-do. 
Table 3. Comparison of the results of the TB screening project conducted in the Jeollanam-do region: "annual TB screening program for vulnerable populations" and "2017 TB screening for the elderly"

\begin{tabular}{|lrrrrr|}
\hline & \multicolumn{3}{c}{ TB screening program for vulnerable population* } & \multirow{2}{*}{ 2017 TB screening for elderly } \\
\cline { 2 - 5 } & $\mathbf{2 0 1 4}$ & $\mathbf{2 0 1 5}$ & $\mathbf{2 0 1 6}$ & $\mathbf{2 0 1 7}$ & 12,402 \\
\hline Total screening subjects & 13,649 & 14,513 & 10,453 & 8,105 & 211 \\
Suspected TB cases & 388 & 418 & 261 & 120 & 16 \\
\hline Notified TB cases & 10 & 13 & 3 & 5 & 0.13 \\
Case detection rate & 0.07 & 0.09 & 0.03 & 0.06 & 775 \\
\hline Number needed to screen & 1,365 & 1,116 & 3,484 & 1,621 & \\
\hline
\end{tabular}

*Korean National Tuberculosis Association (2017). Korean National Tuberculosis Association annual report 2016. Seoul: Korean National Tuberculosis Association $^{15}$, with permission of Korean National Tuberculosis Association.

TB: tuberculosis.

from those reported nationally, although the pilot project detected a lower number of TB cases. Moreover, this project demonstrated the screening efficiency. Sixteen patients were identified as having TB in this project; the NNS for detecting an active TB case was 775. Compared with this outcome, in a recent TB screening program conducted in the JN area from 2014 to 2017, which targeted vulnerable populations and did not specifically focus on the elderly, the NNS was 1,365, 1,116, $3,484$, and 1,621 for each year, respectively (Table 3$)^{15}$. Therefore, TB screening is more likely to be effective for the elderly population than for vulnerable populations including the elderly, those in long-term care facilities, and immigrants.

The risk of active TB could not be predicted based on the history of TB screening performed within the last 12 months, TB-related symptoms, and other comorbidities. Our results highlight the necessity to perform annual systematic screening among the elderly in intermediate- or low-TB burden countries. A model-based study in high-TB burden settings identified the cost-effectiveness of "the periodic active case finding ${ }^{\prime 16}$. Our study outcomes support the benefit of TBspecific screening to identify active TB cases regardless of recent X-ray screening history (within 12 months). Our results assured that duplicated X-ray screening within a year was not a waste of resources among the elderly. Additionally, annual X-ray screening for active case finding may detect newly progressed active TB cases earlier than passive case finding strategies among elderly populations.

In our study, the sputum collection rate was $85.8 \%(n=181)$ among cases with presumptive TB signs on chest X-rays. Most baseline characteristics did not significantly differ between cases who provided sputum, with the exception of those who did not provide sputum (11.1\% vs. $26.7 \%$ ). We could not conclude if the sputum collection rate in our study was acceptable, because previous studies also reported a wide range of sputum collection rates ${ }^{17-19}$. However, innovative tools to include all cases with presumptive TB symptoms should be incorporated into diagnostic evaluations to ensure that no active TB cases are missed.
This study was subject to several limitations. First, there were unmeasured variables such as: participants' socioeconomic status including educational levels, occupation, and household incomes; marital status such as living alone or living with other family members; accessibility of nearby health facilities. Second, final treatment outcomes were not measured in the current study, although cascade analysis is typically used to follow patients until they have final treatment outcomes. Since this study was conducted over the first year of the pilot project, we were unable to report on final treatment outcomes. After the completion of the 4-year project, the investigators plan to report on the final treatment outcomes. Despite this limitation, it is also important to report the case detection rates as one of outcome measures for active case finding interventions.

Mobile screening is an older recommendation in TB management policies; it was not previously recommended by the World Health Organization due to the low yield and relatively high cost associated with case detection ${ }^{20}$. However, the global policy was modified in the End TB Strategy suggesting that active case finding should be prioritized among high-risk group $^{21}$. In the Korean context, the elderly had the highest burden of TB and mortality among all TB cases. Therefore, systematic screening regardless of their TB-related symptoms should be highlighted to prevent additional transmission and to generate favorable treatment outcomes through early case detection. To achieve these results, we identified that several challenges were eliminated in the field. These challenges included duplicate X-ray screening conducted within a year and difficulty mobilizing the elderly participants in the community setting. Our pilot project showed that such challenges could be mitigated and thus they may support the necessity of systematic screening in programmatic settings. Moreover, we expect that early detection of cases without TB-related symptoms and other comorbidities is likely to decrease TBassociated mortality in the elderly. A review involving systematic screening reported the detection of less severe TB cases; however, treatment outcomes remained unchanged from 
those cases detected using passive case detection ${ }^{22}$. However, if the scope of systematic screening is limited to the elderly population, an improvement in treatment outcomes might be achieved because delayed diagnosis may cause more harm to older than younger patients ${ }^{23}$. Therefore, future studies should focus on improving treatment outcomes among the elderly in systematic screening projects.

This study identified that community-based systematic TB screening among the elderly population was recommendable; the prevalence rate did not differ from that previously reported by national TB notification data among the same age groups. Considering the burden of TB among the elderly population, early detection of TB should be prioritized as one of the crucial issues in the national TB program.

\section{Authors' Contributions}

Conceptualization: Choi H, Kim HJ. Methodology: Choi H. Software: Oh KH. Validation: Kim HJ. Formal analysis: Choi H, Kim H, Oh KH. Investigation: Oh HW. Data curation: Kim H. Writing - original draft preparation: Kim H. Writing - review and editing: Choi H, Kim HJ, Oh KH, Oh HW. Approval of final manuscript: all authors.

\section{Conflicts of Interest}

No potential conflict of interest relevant to this article was reported.

\section{References}

1. World Health Organization. Global tuberculosis report 2017 [Internet]. Geneva: World Health Organization; 2017 [cited 2018 Jul 30]. Available from: http://www.who.int/tb/publications/global_report/en/.

2. Rajagopalan S. Tuberculosis and aging: a global health problem. Clin Infect Dis 2001;33:1034-9.

3. Korea Centers for Disease Control and Prevention. Infectious diseases surveillance yearbook, 2016 [Internet]. Cheongju: Korea Centers for Disease Control and Prevention; 2017 [cited 2018 Jul 30]. Available from: https://www.cdc.go.kr/CDC/ info/CdcKrInfo0302.jsp?menuIds=HOME001-MNU1132MNU1138-MNU0038.

4. Korea Centers for Disease Control and Prevention. Annual report on the notified tuberculosis in Korea, 2016 [Internet]. Cheongju: Korea Centers for Disease Control and Prevention; 2018 [cited 2018 Jul 30]. Available from: https://www.cdc. go.kr/CDC/info/CdcKrInfo0302.jsp?menuIds=HOME001MNU1132-MNU1138-MNU0038.

5. Ananthakrishnan R, Kumar K, Ganesh M, Kumar AM, Krish- nan N, Swaminathan S, et al. The profile and treatment outcomes of the older (aged 60 years and above) tuberculosis patients in Tamilnadu, South India. PLoS One 2013;8:e67288.

6. Bennett DE, Courval JM, Onorato I, Agerton T, Gibson JD, Lambert L, et al. Prevalence of tuberculosis infection in the United States population: the national health and nutrition examination survey, 1999-2000. Am J Respir Crit Care Med 2008;177:348-55.

7. Ohmori M, Ishikawa N, Yoshiyama T, Uchimura K, Aoki M, Mori T. Current epidemiological trend of tuberculosis in Japan. Int J Tuberc Lung Dis 2002;6:415-23.

8. Tocque K, Bellis MA, Tam CM, Chan SL, Syed Q, Remmington T, et al. Long-term trends in tuberculosis: comparison of age-cohort data between Hong Kong and England and Wales. Am J Respir Crit Care Med 1998;158:484-8.

9. Wang L, Zhang H, Ruan Y, Chin DP, Xia Y, Cheng S, et al. Tuberculosis prevalence in China, 1990-2010; a longitudinal analysis of national survey data. Lancet 2014;383:2057-64.

10. Park YK, Park YS, Na KI, Cho EH, Shin SS, Kim HJ. Increased tuberculosis burden due to demographic transition in Korea from 2001 to 2010. Tuberc Respir Dis 2013;74:104-10.

11. Kim JH, Yim JJ. Achievements in and challenges of tuberculosis control in South Korea. Emerg Infect Dis 2015;21:1913-20.

12. Kim CH, Choi MH, No MS, Gwak MJ, Han JH, Ha ID, et al. Epidemiologic characteristics of incidence, death and spread of TB in South Korea [Internet]. Cheongju: Korea Centers for Disease Control and Prevention; 2016 [cited 2018 Oct 1]. Available from: http://www.prism.go.kr/homepage/entire/ retrieveEntireDetail.do?pageIndex=2\&research_id=1351000201500304\&leftMenuLevel=160\&cond_research_name= \%EA\%B2\%B0\%ED\%95\%B5\&cond_research_start_date= \&cond_research_end_date=\&pageUnit=10\&cond_order=3.

13. World Health Organization. Systematic screening for active tuberculosis: an operational guide [Internet]. Geneva: World Health Organization; 2015 [cited 2018 Jul 30]. Available from: http://www.who.int/tb/publications/systematic_screening/ en/.

14. Choi HJ, Kim HJ, Oh KH, Chung HJ, Kim HY, Kim MH, et al. Development of a pilot model for tuberculosis management and care for socioeconomically vulnerable regions and groups [Internet]. Cheongju: Korea Centers for Disease Control and Prevention; 2017 [cited 2018 Oct 1]. Available from: http://www.prism.go.kr/homepage/entire/retrieveEntireDetail.do?research_id=1351000-201600218.

15. Korean National Tuberculosis Association. Korean National Tuberculosis Association annual report 2016. Seoul: Korean National Tuberculosis Association; 2017.

16. Dodd PJ, White RG, Corbett EL. Periodic active case finding for TB: when to look? PLoS One 2011;6:e29130.

17. Lorent N, Choun K, Thai S, Kim T, Huy S, Pe R, et al. Community-based active tuberculosis case finding in poor urban settlements of Phnom Penh, Cambodia: a feasible and effective strategy. PLoS One 2014;9:e92754. 
18. Pronyk PM, Joshi B, Hargreaves JR, Madonsela T, Collinson MA, Mokoena O, et al. Active case finding: understanding the burden of tuberculosis in rural South Africa. Int J Tuberc Lung Dis 2001;5:611-8.

19. Prasad BM, Satyanarayana S, Chadha SS, Das A, Thapa B, Mohanty S, et al. Experience of active tuberculosis case finding in nearly 5 million households in India. Public Health Action 2016;6:15-8.

20. World Health Organization. The Stop TB strategy: building on and enhancing DOTS to meet the TB-related Millennium Development Goals. WHO/HTM/TB [Internet]. Geneva: World Health Organization; 2016 [cited 2018 Jul 30]. Available from: http://apps.who.int/iris/handle/10665/69241.
21. World Health Organization. Implementing the end TB strategy: the essentials. WHO/HTM/TB [Internet]. Geneva: World Health Organization; 2015 [cited 2018 Jul 30]. Available from: http://www.who.int/tb/publications/2015/The_Essentials_ to_End_TB/en/.

22. Kranzer K, Afnan-Holmes H, Tomlin K, Golub JE, Shapiro AE, Schaap A, et al. The benefits to communities and individuals of screening for active tuberculosis disease: a systematic review. Int J Tuberc Lung Dis 2013;17:432-46.

23. Lin YS, Yen YF. Determinants of mortality before start of and during tuberculosis treatment among elderly patients: a population-based retrospective cohort study. Age Ageing 2015;44:490-6. 